\title{
Charmed matter
}

\author{
G. Krein \\ Instituto de Física Teórica, Universidade Estadual Paulista \\ Rua Dr. Bento Teobaldo Ferraz, 271 - Bloco II, 01140-070 São Paulo, SP - Brazil
}

\begin{abstract}
In the near future experiments will be able to produce charmed hadrons almost at rest in the interior of an atomic nucleus. One of the most exciting perspectives is the possibility of studying charmonium in a dense medium. In the present communication we present results of a study that explores the possibility that $J / \Psi$ might be bound in a large nucleus through the excitation of intermediate states of $D$ and $D^{*}$ mesons. We also present results of a recent prediction for the production of $\bar{\Lambda}_{c}^{-} \Lambda_{c}^{+}$in proton-antiproton annihilation experiments.
\end{abstract}

Keywords: Charmonium in matter, heavy-light mesons, proton-antiproton annihilation

PACS: $24.85 .+\mathrm{p}, 21.85 .+\mathrm{d}, 21.65 \mathrm{Jk}, 21.65 . \mathrm{Qr}, 25.43 .+\mathrm{t}, 13.75 . \mathrm{Cs}$

\section{INTRODUCTION}

The possibility in the near future of implanting charmed hadrons almost at rest in the interior of an atomic nucleus will open a new era for hadronic matter research. One of the most exciting perspectives is the study of charmonium in a dense medium. This is exciting because the interactions of a charmonium state with the nucleons of the medium are governed by multiple-gluon processes. Since charmonium and ordinary hadrons (formed by $u$ and $d$ quarks) have no quarks in common, their low-energy interactions must proceed via direct multiple-gluon exchanges, like color van der Waals forces [1, 2], or via the creation from the vacuum of light quark-antiquark pairs leading to interactions mediated by heavy-light states like $D$ mesons $[3,4]$. Such processes are distinctive of nonperturbative QCD and are not yet understood. Charmonium can be implanted in a nucleus in experiments of electron scattering off nuclei or in experiments of antiproton annihilation on nuclei. In the next Section we present results of an ongoing study [5] of the contributions of $D$ and $D^{*}$ intermediate states for the $J / \Psi$ self-energy in nuclear matter. In this first study, medium effects are included by coupling the intermediate mesons to nuclear mean fields $\sigma$ and $\omega$.

One of the major difficulties in the study of charmed hadrons in medium is the lack of experimental information on the interactions in free space. Knowledge of the freespace interactions is an important prerequisite for constraining models and assessing medium effects on charmed hadrons. In the absence of such a knowledge one way to make progress is to use symmetry arguments and analogies with processes that share similarities with those of interest and are constrained by data. Such an attitude was taken in recent studies of the $D N$ interaction $[6,7]$ and $\bar{\Lambda}_{c}^{-} \Lambda_{c}^{+}$production in proton-antiproton annihilation [8]. In these, previous experience with successful models for processes involving strangeness, like $K N$ scattering and antiproton-proton production of $\Lambda \bar{\Lambda}$, was used to make predictions for the corresponding processes involving charmed hadrons.

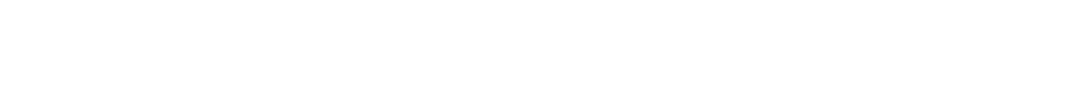


We present here the results of a recent study of the production of $\bar{\Lambda}_{c}^{-} \Lambda_{c}^{+}$baryons in proton-antiproton annihilation experiments. Predictions for such reactions are important for assessing orders of magnitudes of cross sections, which is extremely important for guiding planned experiments.

\section{$J / \Psi$ BINDING TO NUCLEAR MATTER}

Initially we discuss results of a recent study [5] of the $J / \Psi$ self-energy in nuclear matter considering the loop of $D$ mesons as shown in Fig. 1. The effects of the nuclear medium on the intermediate $D$ and $D^{*}$ mesons are calculated with the quark-mesoncoupling (QMC) model [9]. The QMC model is a relativistic field theory model, where effective scalar $(\sigma)$ and vector $(\omega$ and $\rho)$ meson mean fields are coupled directly to the light $u$ and $d$ quarks confined in a bag (or by a potential [10]). A very reasonable description of saturation properties of nuclear matter and of single-particle energy levels of finite nuclei is achieved in this model - for a review see Ref. [11].

The contributions from $D D, D D^{*}$ and $D^{*} D^{*}$ loops to the $J / \Psi$ self-energy are calculated using effective Lagrangians. Let us consider first the $D D$ loop. The Lagrangian density for the $J / \Psi-D D$ vertex used in the calculation is given by

$$
\mathscr{L}_{\psi D \bar{D}}=i g_{\psi D D} \psi^{\mu}\left[\bar{D}\left(\partial_{\mu} D\right)-\left(\partial_{\mu} \bar{D}\right) D\right]
$$

where $\psi$ is the field corresponding to $J / \Psi$. We are interested in the difference between the in-medium and vacuum masses of $J / \Psi, \Delta m=m_{\psi}^{*}-m_{\psi}$, with $m_{\psi}^{2}=\left(m_{\psi}^{0}\right)^{2}+\Sigma\left(k^{2}=\right.$

$\left.m_{\psi}^{2}\right)$, where $m_{\psi}^{0}$ is the bare mass and $\Sigma\left(k^{2}\right)$ is the $J / \Psi$ self-energy. The in-medium mass $m_{\psi}^{*}$ is given by the same expression as for $m_{\psi}$, with the self-energy calculated with medium-modified $D$ mesons.

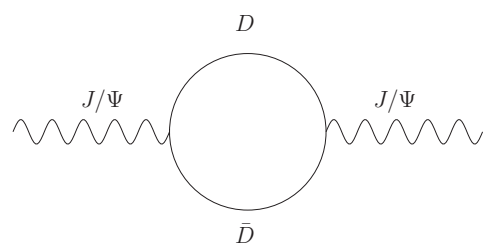

FIGURE 1. $D D$-loop contribution to the $J / \Psi$ self-energy. There are also $D D^{*}$ and $D^{*} D^{*}$ contributions.

A calculation similar to the one here was performed some time ago in Ref. [4]. Using a gauged $J / \Psi D \bar{D}$ effective Lagrangian, the authors of Ref. [4] found that the mass of $J / \Psi$ in medium is larger than in free space, indicating that $J / \Psi$ would not be bound to nuclear matter. We believe that the repulsive result for the self-energy is due to the use of a gauged Lagrangian. The difference between Eq. (1) and the gauged Lagrangian of Ref. [4] is a term $2 g_{\psi D D}^{2} \psi^{\mu} \psi_{\mu} \bar{D} D$. For equal masses $m_{D}$ for the charged and neutral $D$ mesons, the self-energy is given by (for $J / \Psi$ at rest)

$$
\Sigma^{\psi}\left(m_{\psi}^{2}\right)=-\frac{1}{3 \pi^{2}} g_{\psi D D}^{2} \int_{0}^{\infty} d q \frac{q^{2}}{\omega_{D}(q)}\left[\frac{q^{2}}{\omega_{D}^{2}\left(q^{2}\right)-m_{\psi}^{2} / 4}-\xi\right] F\left(q^{2}\right),
$$


where $\xi=0$ for the Lagrangian in Eq. (1) and $\xi=3$ for the gauged Lagrangian of Ref. [4], $\omega_{D}(q)=\left(q^{2}+m_{D}^{2}\right)^{1 / 2}$, and $F\left(q^{2}\right)$ is a form factor. One sees that the integrand changes sign as $q$ increases, being negative for $q$ small and the overall sign depends then on how much the form factor cuts off the high momentum part of the integrand.

The additional $D D^{*}$ and $D^{*} D^{*}$ contributions are calculated with the following Lagrangian densities,

$$
\begin{aligned}
\mathscr{L}_{\psi D D^{*}}= & \frac{g_{\psi D D^{*}}}{m_{\psi}} \varepsilon_{\alpha \beta \mu v}\left(\partial^{\alpha} \psi^{\beta}\right)\left[\left(\partial_{\mu} \bar{D}^{* v}\right) D+\bar{D}\left(\partial_{\mu} D^{* v}\right)\right], \\
\mathscr{L}_{\psi D^{*} D^{*}}= & i g_{\psi D^{*} D^{*}}\left\{\psi^{\mu}\left[\left(\partial_{\mu} \bar{D}^{* v}\right) D_{v}^{*}-\bar{D}^{* v}\left(\partial_{\mu} D_{v}^{*}\right)\right]\right. \\
& +\left[\left(\partial_{\mu} \psi^{v}\right) \bar{D}_{v}^{*}-\psi^{v}\left(\partial_{\mu} \bar{D}_{v}^{*}\right)\right] D^{* \mu} \\
& \left.+\bar{D}^{* \mu}\left[\psi^{v}\left(\partial_{\mu} D_{v}^{*}\right)-\left(\partial_{\mu} \psi^{v}\right) D_{v}^{*}\right]\right\} .
\end{aligned}
$$

Explicit expressions for the corresponding self-energies will be presented elsewhere [5].

TABLE 1. In medium $J / \Psi$ mass $m_{\psi}^{*}, \Delta m$ and individual loop contributions to the $J / \Psi$ selfenergy at nuclear matter density, for different values of the cutoff $\Lambda$. All quantities are in $\mathrm{MeV}$.

\begin{tabular}{ccc|ccc}
\hline$\Lambda$ & $m_{\psi}^{*}$ & $\Delta m$ & $D D$ & $D D^{*}$ & $D^{*} D^{*}$ \\
\hline 1000 & 3081 & -16 & -3 & -2 & -11 \\
1500 & 3079 & -18 & -3.5 & -2.5 & -12 \\
2000 & 3077 & -20 & -4 & -3 & -13 \\
3000 & 3072 & -24 & -6.5 & -5 & -12.5
\end{tabular}

For the form factor we use a dipole at each vertex parametrized by a cutoff $\Lambda$. We fix the vacuum values of the masses to $m_{\psi}=3096.9 \mathrm{MeV}$ and $m_{D}=1867.2 \mathrm{MeV}, m_{D^{*}}=$ 2008.6 MeV, and determine $m_{\psi}^{0}$ for different values of $\Lambda$. For the coupling constants, we use the values extracted in Ref. [12], namely $g_{\psi D D}=g_{\psi D D^{*}}=g_{\psi D^{*} D^{*}}=7.64$. For the moment we neglect the widths of the $D$ mesons and include only the effects of the nuclear mean fields on the masses of these mesons. As said above, we use the predictions based on the QMC model for the in-medium masses, given in Ref. [13]. In Table 1 we present our results at nuclear matter density. Specifically, we present results for the inmedium mass of $m_{\psi}^{*}, \Delta m$ and the individual loop contributions from $D D, D D^{*}$ and $D^{*} D^{*}$ intermediate states.

Considering $\Delta m_{\psi}$ as a potential, would a mass shift of the order of $10-20 \mathrm{MeV}$, as shown in Table 1 , be enough to bind a $J / \Psi$ to a large nucleus? A rough estimate can be made as follows. From elementary quantum mechanics, one knows that for an attractive spherical well of radius $a$ and depth $V_{0}$, the condition for the existence of an $s$-wave bound state of a particle of mass $m$ is $V_{0}>\pi^{2} \hbar^{2} / 8 m a^{2}$. Using for $m=m_{\psi}$ and $a=5 \mathrm{fm}$, one obtains $V_{0}>1 \mathrm{MeV}$. Therefore, it seems that the perspectives of capturing a $J / \Psi$ in a large nucleus are quite favorable.

Of course, many issues remain to be investigated. The most important ones are the consideration of the widths of the $D$ mesons, that tend to diminish the binding, and the fact that $J / \Psi$ will most likely not be produced at rest in the interior of a nucleus and therefore the self-energies should be calculated for a moving $J / \Psi$. 


\section{PREDICTIONS FOR $\bar{p} p \rightarrow \bar{\Lambda}_{c}^{-} \Lambda_{c}^{+}$}

As mentioned, the absence of experimental information on the low energy interactions of charmed hadrons with nucleons in free space makes it difficult to consider in-medium properties of these interactions. Moreover, cross sections for production of charmed hadrons on nucleons at low energies are also not known experimentally. Here we present a recent prediction [8] for the reaction $\bar{p} p \rightarrow \bar{\Lambda}_{c}^{-} \Lambda_{c}^{+}$, which is one of the planned experiments for FAIR at GSI. This reaction is somewhat simpler than $\bar{p} p \rightarrow \bar{D} D$, which is more relevant for the $J / \Psi$ problem studied in the previous Section, because of the many channels that can be open in the final states in this last reaction. Our strategy for studying the reaction $\bar{p} p \rightarrow \bar{\Lambda}_{c}^{-} \Lambda_{c}^{+}$is similar to our recent work on the $D N$ system [6,7], which was based on a hybrid model for the $K N$ system that uses meson-baryon dynamics at long distances and quark-gluon dynamics at short distances [14, 15]. Specifically, we build on the Jülich coupled channel approach $[16,17]$ for the hyperon production reaction $\bar{p} p \rightarrow \bar{\Lambda} \Lambda$, which takes into account rigorously the effects of the initial $(\bar{p} p)$ and final $(\bar{\Lambda} \Lambda)$ state interactions. In this model, the microscopic strangeness production process and the elastic parts of the interactions in the initial $(\bar{p} p)$ and final $(\bar{\Lambda} \Lambda)$ states are described by meson exchanges, while annihilation processes are accounted for by phenomenological optical potentials. A reasonably good overall description is obtained for the available data on total and differential cross-sections and spin observables.

The basic ingredients of the original Jülich coupled channel approach $[16,17]$ are as follows. The transition amplitude is obtained from the solution of a coupled-channel Lippmann-Schwinger equation, with a driving potential $V$ which is a $2 \times 2$ matrix in channel space. The diagonal potentials are given by the sum of an elastic part and an annihilation part. The elastic part of the $\bar{N} N$ potential is deduced (via G-parity transform) from a simple, energy-independent one-boson-exchange $N N$ potential and a phenomenological spin-, isospin-, and energy-independent optical potential is added to take into account annihilation. In addition, in order to take into account the absorptive part of the $\bar{N} N$ interaction in the initial state, we considered several variants of the $\bar{N} N$ potential - for details see Ref. [8]. The interaction in the final $\bar{\Lambda}_{c}^{-} \Lambda_{c}^{+}$state is assumed to be the same as the one in $\bar{\Lambda} \Lambda$, i.e. the elastic part of the interaction is given by the isospin-zero $\sigma$ and $\omega$ exchanges and the annihilation part is again parameterized by an optical potential which contains central, spin-orbit and tensor components [16]. Finally, the transition potential from $\bar{p} p$ to $\bar{\Lambda}_{c}^{-} \Lambda_{c}^{+}$is given by $t$-channel $D$ and $D^{*}$ exchanges and their explicit expressions are the same as for $K$ and $K^{*}$; they are of the generic form

$$
V^{p \bar{p} \rightarrow \bar{\Lambda}_{c}^{-} \Lambda_{c}^{+}}(t) \sim \sum_{M=D, D^{*}} g_{N \Lambda_{c} M}^{2} \frac{F_{N \Lambda_{c} M}^{2}(t)}{t-m_{M}^{2}},
$$

where $g_{N \Lambda_{c} M}$ are coupling constants and $F_{N \Lambda_{c} M}^{2}(t)$ are form factors. While the couplings are fixed by assuming $S U(4)$ symmetry, the cutoff $\Lambda$ in the vertex form factors larger than in the kaon case, namely $\Lambda=3 \mathrm{GeV}$, at the $N \Lambda_{c} D$ as well as at the $N \Lambda_{c} D^{*}$ (we actually have explored the sensitivity of the results to variations in $\Lambda$ ). 
With the aim of examining uncertainties, we have also considered a charm-production potential inspired by quark-gluon dynamics, given by [18]

$$
V^{\bar{p} p \rightarrow \bar{\Lambda} \Lambda}(r)=\frac{4}{3} 4 \pi \frac{\alpha}{m_{G}^{2}} \delta_{S 1} \delta_{T 0}\left(\frac{3}{4 \pi\left\langle r^{2}\right\rangle}\right)^{3 / 2} \times \exp \left(-3 r^{2} /\left(4\left\langle r^{2}\right\rangle\right)\right),
$$

where $\alpha / m_{G}^{2}$ is an effective (quark-gluon) coupling strength, $\left\langle r^{2}\right\rangle$ is the quark mean square radius distribution in the $p$ or $\Lambda$, and $S$ and $T$ are the total spin and isospin in the $\bar{p} p$ system. Heuristically, $m_{G} \approx 2 m_{q}$ of the produced quarks and so we used a $\alpha / m_{G}^{2}$ for charm production reduced by the ratio $\left(m_{s} / m_{c}\right)^{2} \approx(550 \mathrm{MeV} / 1600 \mathrm{MeV})^{2} \approx 1 / 9$ as compared to the one for $\bar{p} p \rightarrow \bar{\Lambda} \Lambda$.

Our predictions for the total reaction cross sections for $\bar{p} p \rightarrow \bar{\Lambda}_{c}^{-} \Lambda_{c}^{+}$as a function of the excess energy $p_{l a b}$ are shown in Fig. 2. For values of the parameters of the optical potentials, coupling constants and cutoff values, as well as the way these are fixed, see Ref. [8]. We also show in Fig. 2 results from two calculations in the literature [19, 20]. It is seen that our cross sections are a factor 1000 larger than those of Ref. [19], and a factor 100 larger from those in Ref. [20]. From the experimental perspective, our results are good news, in the sense that they are only a factor 10 to 70 smaller than those for $\bar{\Lambda} \Lambda$.

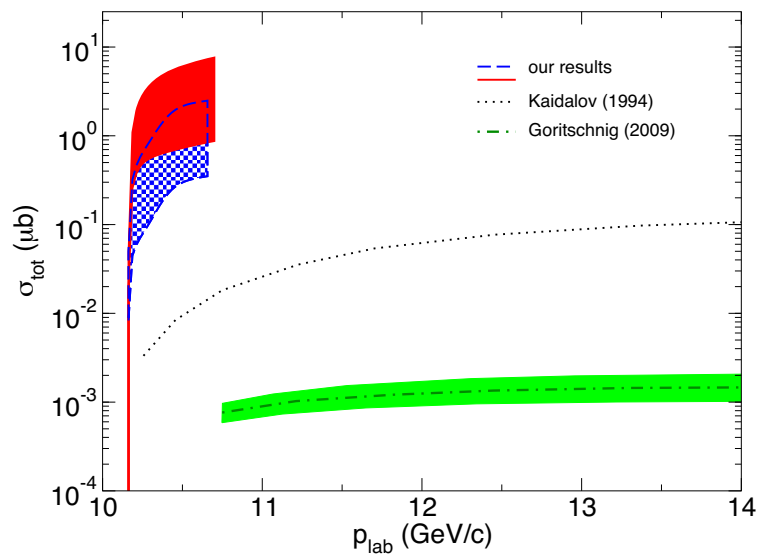

FIGURE 2. Total cross sections for $\bar{p} p \rightarrow \bar{\Lambda}_{c}^{-} \Lambda_{c}^{+}$as a function of the excess energy $p_{\text {lab }}-$ figure from Ref. [8]. The dark (red) shaded band (blue grid) is obtained with a meson-exchange (quark-gluon) transition potential. The dotted curve is the result from Ref. [20] while the dash-dotted curve and the corresponding (green) band is from Ref. [19].

\section{CONCLUSIONS}

We presented results of a study [5] for the existence of an exotic nuclear bound state in which a charmonium state produced close to threshold is captured in matter. Attraction of $J / \Psi$ to nuclear matter is achieved via the excitation of an intermediate state of $D$ and 
$D^{*}$ mesons. Our results indicate that when the masses of the $D$ and $D^{*}$ mesons are made density dependent according to the QMC model, sufficient attraction for the formation of a $J / \Psi$-nucleus bound state is achieved.

We have also shown results of a recent study of the production of $\bar{\Lambda}_{c}^{-} \Lambda_{c}^{+}$in protonantiproton annihilation [8]. The calculations are based on a meson-exchange framework in close analogy to an earlier study on $\bar{p} p \rightarrow \bar{\Lambda} \Lambda$, using SU(4) symmetry for fixing unknown coupling constants. Our prediction for the $\bar{\Lambda}_{c}^{-} \Lambda_{c}^{+}$production cross sections are in the order of 1 to $7 \mu b$, just 10-70 times smaller than the corresponding cross sections for $\bar{\Lambda} \Lambda$. The use of a quark model based transition potential, instead of $D$ and $D^{*}$ meson exchanges, leads to essentially the same results.

\section{ACKNOWLEDGMENTS}

The author would like to thank J. Haidenbauer, A.W. Thomas and K. Tsushima for the collaboration on the work presented here. Work partially financed by FAPESP and CNPq (Brazilian agencies).

\section{REFERENCES}

1. M. E. Peskin, Nucl. Phys. B 156, 365 (1979).

2. S. J. Brodsky, I. A. Schmidt and G. F. de Teramond, Phys. Rev. Lett. 64, 1011 (1990).

3. S. J. Brodsky and G. A. Miller, Phys. Lett. B 412, 125 (1997).

4. S. H. Lee and C. M. Ko, Phys. Rev. C 67, 038202 (2003).

5. G. Krein, A.W. Thomas and K. Tsushima, in preparation.

6. J. Haidenbauer, G. Krein, U. G. Meißner and A. Sibirtsev, Eur. Phys. J. A 33, 107 (2007).

7. J. Haidenbauer, G. Krein, U.-G. Meißner and A. Sibirtsev, Eur. Phys. J. A 37, 55 (2008).

8. J. Haidenbauer and G. Krein, Phys. Lett. B 687, 314 (2010).

9. P. A. M. Guichon, Phys. Lett. B 200, 235 (1988).

10. M.E. Bracco, G. Krein and M. Nielsen, Phys. Lett. B 432, 258 (1998).

11. K. Saito, K. Tsushima and A. W. Thomas, Prog. Part. Nucl. Phys. 58, 1 (2007).

12. Z. w. Lin and C. M. Ko, Phys. Rev. C 62, 034903 (2000).

13. A. Sibirtsev, K. Tsushima and A. W. Thomas, Eur. Phys. J. A 6, 351 (1999).

14. D. Hadjimichef, J. Haidenbauer and G. Krein, Phys. Rev. C 66, 055214 (2002).

15. J. Haidenbauer and G. Krein, Phys. Rev. C 68, 052201 (2003).

16. J. Haidenbauer et al. , Phys. Rev. C 45, 931 (1992).

17. J. Haidenbauer, K. Holinde, V. Mull and J. Speth, Phys. Rev. C 46, 2158 (1992).

18. M. Kohno and W. Weise, Phys. Lett. B 179, 15 (1986).

19. A. T. Goritschnig, P. Kroll and W. Schweiger, Eur. Phys. J. A 42, 43 (2009).

20. A. B. Kaidalov and P. E. Volkovitsky, Z. Phys. C 63, 517 (1994). 
Copyright of AIP Conference Proceedings is the property of American Institute of Physics and its content may not be copied or emailed to multiple sites or posted to a listserv without the copyright holder's express written permission. However, users may print, download, or email articles for individual use. 